\title{
Which impact for proton pump inhibitors in SARS-CoV-2 pneumonia
}

\author{
Grazia Mazzeo ${ }^{1,2,3}$, Luigi Aronne ${ }^{1,2}$, Domenica Francesca Mariniello ${ }^{1,2}$, Valentino Allocca ${ }^{1,2}$, \\ Maria Ilaria Palma ${ }^{1,2}$, Francesco Saverio Cerqua ${ }^{1,2}$, Carlo Iadevaia ${ }^{1,2}$, Adriano Costigliola ${ }^{1,2}$, \\ Roberto Parrella ${ }^{4}$, Andrea Bianco ${ }^{1,2,3}$, Vanvitelli/Monaldi COVID Group*
}

${ }^{1}$ Department of Translational Medical Sciences, University of Campania L. Vanvitelli, Monaldi Hospital, Naples; ${ }^{2}$ Department of Pneumology and Oncology, AORN dei Colli, Monaldi Hospital, Naples; ${ }^{3}$ Vanvitelli COVID-19 Unit, Infectious Disease Department, University of Campania L. Vanvitelli, Naples; ${ }^{4}$ U.O.C. Respiratory Infectious Diseases, AORN dei Colli, Cotugno Hospital, Naples, Italy

\begin{abstract}
Identification of risk factors for severe outcome of SARS-CoV2 infection is an important issue in COVID-19 management. Much attention has been focused on comorbidities as well as drugs taken by patients. Usage of proton pump inhibitors (PPIs) appears to potentially influence disease course. These drugs are known to reduce stomach acid and also modulate the immune system. Their use, prior to and during COVID-19 infection, seems to predispose to the development of more severe pneumonia and therefore to a greater risk of mortality. Instead, the use of Histamine receptor 2 antagonists (H2RAs) seems to be associated with a better outcome in patients with COVID-19, in terms of symptoms, risk of intubation and death. As PPIs are essential for treatment of many disorders, usage of these drugs should be balanced considering the
\end{abstract}

Correspondence: Grazia Mazzeo, Department of Translational Medical Sciences, University of Campania L. Vanvitelli, Monaldi Hospital, 80131 Naples, Italy. E-mail: graziamazz11@gmail.com

Key words: COVID-19; SARS-CoV-2; proton pump inhibitors; histamine receptor 2 antagonists, pneumonia.

Contributions: All the authors made a substantive intellectual contribution, have read and approved the final version of the manuscript and agreed to be accountable for all aspects of the work.

Conflict of interest: The authors declare that they have no competing interests, and all authors confirm accuracy.

Availability of data and material: all the sources cited were searched on online databases, mainly PubMed.

Received for publication: 14 February 2021.

Accepted for publication: 8 June 2021.

${ }^{\circ}$ Copyright: the Author(s), 2021

Licensee PAGEPress, Italy

Monaldi Archives for Chest Disease 2021; 91:1803

doi: 10.4081/monaldi.2021.1803

This article is distributed under the terms of the Creative Commons Attribution Noncommercial License (by-nc 4.0) which permits any noncommercial use, distribution, and reproduction in any medium, provided the original author(s) and source are credited. benefits and risk ratio, in order to guarantee their correct use for the necessary time. It remains to be clarified whether the detrimental effects, in terms of COVID-19 severe outcome, are due to PPIs or to the underlying disease for which they are administered. New controlled-randomized trials are required to better understand their impact in SARS-CoV-2 infections.

\section{Introduction}

Severe acute respiratory syndrome coronavirus 2 was declared a pandemic in March 2020. The virus causes coronavirus disease 2019 (COVID-19) which can range from asymptomatic or mild infection to multiorgan dysfunction (MOD), acute respiratory distress syndrome (ARDS), and death [1,2]. Symptomatically, the virus can cause cough, fever, myalgia, dyspnea, altered smell or taste, pharyngodynia, diarrhoea, vomiting and nausea [3-5]. Identification of the risk factors that could influence the course of this disease is important regarding decisions involving public health recommendations and clinical interventions. There are several confirmed risk factors of COVID-19, including old age [6], chronic pulmonary disease and smoking, cardiovascular disease, chronic kidney disease, diabetes mellitus and obesity, malignancy $[7,8]$. There is a growing interest in how exposure to certain medicines affects the risk of SARS-CoV-2 infection [9]. Several medications including anticoagulant and metformin are associated with a reduction in poor outcomes from COVID-19, while other medications do not appear to alter outcomes of COVID-19 infections such as ACE inhibitors, angiotensin II receptor blockers (ARB) and statins [10-12].

Proton pump inhibitors are among the top ten most widely used drugs in the world; their use may increase the risk of pneumonia [13] and have been linked to side effects including bone fracture, chronic kidney disease, and gastrointestinal (GI) infections, among others [14]. PPIs are among the most used drugs by general population for their efficacy to improve symptoms like dyspepsia and GERD symptoms as well as for their relatively low cost [15].

\footnotetext{
*Vanvitelli/Monaldi COVID Group:

Adriano Cristinziano, Carolina Delle Donne, Cecilia Calabrese, Fabio Perrotta, Filippo Scialò, Francesco Lassandro, Gennaro Mazzarella, Giorgio Paoli, Leonardo De Luca, Maria Galdo, Miriam Buonincontro, Roberta Cianci, Rosalba Donizzetti, Stefano Sanduzzi Zamparelli, Tullio Valente, Vito D'Agnano, Vittorio Bisogni.
} 
In clinical practice PPI's use is not uncommon in patients receiving corticosteroids. Based on the hypothesis that use of PPIs in COVID 19 may affect clinical outcomes, this review explores the actual knowledge on the mechanisms implicated in the balance between risks and benefits associated with proton pump inhibitors usage in COVID patients leading to severe outcomes.

\section{Methods}

Using online databases, we carried out a systematic literature review of the clinical outcomes of COVID-19 associated with the use of proton pump inhibitors (PPI) and/or histamine 2 receptor antagonists (H2RA), particularly famotidine. Key articles were retrieved mainly from PubMed, Google Scholar and Embase using the terms "acid suppressive therapy", "antiacid", "proton pump inhibitors", "pantoprazole", "histamine receptor 2 antagonists", "H2RAs", "famotidine" and "COVID 19" or "SARS-CoV-2" as keywords for our search. We included scientific publications from April 2020 to November 2020. Only publications focusing on clinical outcomes of COVID-19 and treatments with PPIs or H2RAs, such as Famotidine, were eligible for inclusion.

Luxenburger et al.'s work is the first to provide data in support of our hypothesis, as patients with COVID-19 treated regularly with PPIs before hospitalization were found to have a significantly higher mortality index [16].

Meta-analysis by Hariyanto et al., which included 6 studies [1618 ] with a total of 5884 COVID-19 patients, showed PPI usage is significantly associated with an increased risk of severity (RR 1.35 , 95\%CI, 1.11-1.63; $\mathrm{p}=0.003$ ) and mortality (RR 1.72, 95\% CI, 1.02$2.89, \mathrm{p}=0.04$ ) for COVID-19 infection.

A nationwide cohort study on severe clinical outcomes of COVID-19 associated with proton pump inhibitors by Lee et al. [18] concluded that current use of PPIs was related to increased risk of requirement of oxygen therapy, intensive care unit admission, administration of invasive ventilation or death in patients with COVID-19 infection (aOR 1.63; 95\%CI, 1.03 to 2.53) while the relationship with past PPI use was non-significant (aOR 0.98; $95 \% \mathrm{CI}, 0.39$ to 2.47 ). The current use of PPIs was also related to increased risk for severe outcomes of COVID-19 (aOR 1.79; $95 \%$ CI, 1.30 to 3.10 ), while the relationship with past PPI use was insignificant (aOR 1.18; 95\% CI, 0.27 to 5.13).

\section{Risks and benefits associated with proton pump inhibitors usage}

Based on analysis of available data, the use of proton pump inhibitors (PPIs) seems to be associated with increased risk of severity and mortality from COVID-19.

Several pathogenic mechanics have been proposed to explain this association between PPI use and increased risk of adverse outcomes of COVID-19. PPIs are drugs used in the treatment of gastric acid-related diseases that suppress acid secretion in the gastric lumen [19] through inhibition of $\mathrm{H}+/ \mathrm{K}+$ ATPase. One of the main functions of gastric secretions is to inactivate swallowed microorganisms, thereby inhibiting infectious agents from reaching the intestine; gastric secretions are thus the first line of defense against pathogens. PPI-induced hypochlorhydria impairs the body's proximal defense against ingested bacteria and viruses [14], resulting in insufficient eradication of these pathogens with alteration of various immune-modulatory and anti-inflammatory effects [20].

Prolonged use of PPIs reduces microbial diversity in the gut [21], an effect believed to enable colonization of some enteric pathogens [22]. The profound hypochlorhydric condition can cause an increase in gastric microbiota and small intestinal bacterial overgrowth. The resulting dysbiosis may increase the likelihood of developing enteric infections and sepsis which could complicate the disease.

Although the impact of acid suppression on severe acute respiratory syndrome coronavirus 2 (SARS-CoV-2) is unknown thus far, previous data has revealed that $\mathrm{pH}<3$ - the normal $\mathrm{pH}$ of a healthy stomach - impairs the infectivity of the similar severe acute respiratory syndrome coronavirus 1 , whereas less acidic $\mathrm{pH}$ in the range achieved with PPI therapy does not inactivate the virus [23]. Suppression of gastric acid may increase the survival of SARS-CoV-2 in the stomach and increase the ability of the virus to invade the GI epithelial cells.

SARS-CoV-2 can enter the body not only through the respiratory system but also through the GI system [24,25]. SARSCov-2 uses the angiotensin-converting enzyme-2 receptor (ACE2) to gain entry into human cells. Small intestine, kidney, testis, heart muscle, colon, and thyroid gland are the overlapping tissues with the highest level of ACE2 expression, with no expression detected in blood cells [26]. These data explain why people affected by COVID-19 experience gastrointestinal problems and kidney dysfunction [26-28]. ACE2 is widely expressed throughout the intestinal tract [29], enabling rapid invasion and replication within enterocytes [27]. The fecal-oral route has been proposed as one of the potential modes of transmission for COVID-19. Regarding SARS-CoV-2, Xiao et al. provided evidence for gastrointestinal infection and demonstrated that SARS-CoV-2 infects gastrointestinal glandular epithelial cells [25]. Viral RNA was found in rectal swabs, even after nasopharyngeal testing has turned negative, implying a fecal-oral transmission [25], and it is of note that patients with coronavirus disease 2019 (COVID-19) who experience gastrointestinal symptoms are more likely to have severe pneumonia [30].

Once SARS-CoV-2 colonizes the GI tract, it can lead to gastritis, enteritis, and colitis [25,31]. The higher expression of ACE 2 in the GI tract allows higher viral entry to cells [32] resulting in higher viral loads, possibly causing more severe disease due to cytokine storm. Even in respiratory tract disease, individuals with more virus colonization in the stomach due to increased gastric alkalinity caused by PPI administration may be more susceptible to severe courses of COVID-19. Other investigators posit that SARSCoV-2's disruption of the epithelial layer can lead to release of endotoxins and microbial metabolites that subsequently trigger inflammation and cytokine release in distant organs, such as the lungs [24,33]. Inflammatory mediators can elevate platelet count, down-regulate natural anticoagulant mechanisms, facilitate propagation of the coagulant response and impair fibrinolysis [34].

Interestingly, patients with immune-mediated disorders (i.e., rheumatoid arthritis, psoriasis, ankylosing spondylitis and IBDs) who are treated with anticytokine therapy do not appear to be vulnerable to several adverse clinical outcomes of COVID-19 [35]. Anticytokine therapy may have a protective effect against the cytokine storm. In this respect, PPI usage may lead to worse outcomes of COVID-19 resulting from a more intense cytokine storm. In addition, because the gut is the largest immune organ in the body and can host colonies of rapidly replicating SARS-CoV-2 [27], there is concern that the virus could spread beyond the GI tract 
not only causing digestive symptoms but also by seeding infection or promoting inflammation in other organ systems, including the respiratory tract via a "gut-lung axis" [24,33].

Previous research with the Middle East Respiratory Syndrome coronavirus found that pretreating mice with pantoprazole, a PPI, showed "exaggerated" evidence of not only enteric infection but also revealed epithelial degeneration in the small bowel. Notably, the virus was subsequently found to emerge in lung tissue. The authors note that the spread of virus from intestine to lungs indicates "development of sequential respiratory infection" after inoculating the stomach - not the lungs - with a coronavirus that uses the angiotensin-converting enzyme-2 receptor to gain gut entry into the body [36].

\section{Proton pump inhibitors and SARS-CoV-2 respiratory involvement}

The use of acid-suppressive drugs has been associated with an increased risk of pneumonia. A large number of investigations have focused on the biological plausibility of these observations [37]. First, acid-suppressive drugs may increase the risk of pneumonia by inhibiting the secretion of gastric acid allowing bacterial overgrowth and colonization in the upper alimentary tract and subsequent translocation to the lungs by aspiration [37,38]. Second, hydrogen potassium adenosine triphosphatase is present not only in the parietal cells of the stomach, but also in the upper and lower respiratory tract [39]; it is conceivable that inhibiting the proton pump, $\mathrm{pH}$ of the secretions and respiratory flora may be altered, leading to increased risk of pneumonia [40]. Third, in vitro studies have shown that acid-suppressive drugs may impair the function of neutrophils and the activity of natural killer cells [41,42]. Interestingly, the most striking increase in the risk of pneumonia in association with proton pump inhibitors was observed in the first weeks of use. During the first months, when aero-digestive microbiome may be in greatest flux, the risk of pneumonia in association with use of proton pump inhibitors is higher; between 30 and 180 days the risk is attenuated, although still significant [37]. By contrast a systematic review and meta-analysis has shown no increase of community acquired pneumonia (CAP) risk in patients receiving H2RAs, probably due to the weaker acid suppressant activity than PPIs [43]. The first observation in patients with COVID-19 has been made by Luxemburger et al.; the authors suggest that regular PPI therapy before hospital admission is a risk factor for development of secondary infections and is associated with significant higher mortality index [16,44]. Observations from Corsonello et al. show that PPI treatment may facilitate bacteria growth; in this context, when micro aspiration occurs secondary infections increase the risk of acute respiratory distress syndrome and mortality rate from COVID-19 $[45,46]$.

\section{PPIs and immune system}

PPIs can also modulate the immune response by inhibiting neutrophil function. Neutrophils play a significant role in the immune innate system which is the primary defense of the body to fight infection. Inhibition of neutrophil function may impair the ability of the body to eradicate infections and may increase the severity of infections, including COVID-19 infection [47].
PPIs can inhibit the enzymatic activity of dimethylarginine dimethylaminohydrolase (DDAH) which will inhibit the nitric oxide synthase with the promotion of inflammation and thrombosis, resulting in the development of cardiovascular disease. These adverse events from PPIs can also contribute to the development of severe outcomes and mortality from COVID-19 infection [48,49].

As already mentioned, a key deleterious effect of a SARS-CoV2 infection is the immune hyperreaction and cytokine storm [50]. This acute hyper inflammatory response is paramount to the severity of the SARS-CoV-2 infection. Taghizadeh-Hesary and Akbari [51] elegantly explain how SARS-COV-2 may cause a depletion of cellular ATP and a dysfunction of immune cells, and they propose a repletion of cellular ATP to improve the efficiency of the immune system. In line with this, Kouhpayeh et al. [52] propose that SARSCoV-2 activates PARP-2 (poly [ADP-ribose] polymerase 2), which induces a depletion of $\mathrm{NAD}^{+}$and consequently also drains cellular ATP. Scrutiny of this hypothesis reveals that a critical part of the process may have been overlooked. For the synthesis and regeneration of ATP, phosphate and magnesium are required. Interestingly, most risk factors for a severe course of COVID-19 are associated with a deficiency of phosphate and/or magnesium. For instance, type 2 diabetic medications [53,54] and diuretics [55] routinely prescribed for hypertension and obesity diminish magnesium and phosphate. The usage of proton-pump inhibitors has also been linked to a worse outcome in COVID-19 patients; such proton-pump inhibitors are known to diminish intestinal magnesium absorption [18].

\section{The role of comorbidities in SARS-CoV-2 infection}

A possible bias is that the risk of severity and mortality from COVID-19 is increased among persons taking PPIs due to the nature of their disease state, unrelated to the PPI therapy. GERD, a common indication for PPI therapy, may increase directly micro aspiration of gut flora. Laryngopharyngeal reflux disease may be higher than the general population [56] and was commonly prevalent in hospitalized patients with COVID-19 and independently associated with risk of severe or critical infection. Furthermore, individuals with certain comorbidities that increase the risk for severe COVID-19 (e.g., cardiovascular disease, chronic obstructive pulmonary disease, and chronic kidney disease) may also be more likely to take PPIs. However, it is unclear whether these risk factors apply to acquiring COVID-19 in contrast to developing severe COVID-19.

\section{Histamine receptor 2 antagonist and COVID-19}

Histamine receptor 2 antagonists (H2RAs) have also been used for suppression of gastric acid production but cause less hypochloridria than PPIs and do not interact with other medications. Famotidine is a selective blocker of the histamine $\mathrm{H} 2$ receptor. A retrospective cohort study of 1620 hospitalized patients with COVID-19 demonstrated that patients receiving famotidine had statistically significant reductions in both inhospital death and combined death and intubation [57]. In contrast proton pump inhibitor's use was not associated with reduced risk for these outcomes. More recently, a small case series of not hospitalized patients with COVID-19 treated with high-dose oral famotidine documented a marked improvement in symptoms in 
all cases shortly after starting the drug [58]. The mechanism by which famotidine might improve COVID-19 outcomes is currently unknown. One theory, based on earlier reports on the efficacy of famotidine in inhibiting human immunodeficiency virus replication [59], is that famotidine may directly inhibit the SARSCoV-2 virus. Recent studies have however failed to demonstrate any direct inhibitory effect of famotidine on SARS-CoV-2 infection [60]. A second theory identified famotidine as a potential agent capable of binding and inhibiting key SARS-CoV-2 proteases critical to viral replication $[61,62]$, has likewise been discounted [60]. A more recent mechanism that has been postulated is that famotidine's effect is achieved via its antagonism or inverse agonism of the histamine- 2 receptor, inferring that the SARS-CoV-2 infection that results in COVID-19 is at least partially mediated by pathological histamine release and perhaps dysfunctional mast cell activation [60,63]. Preventing the deleterious sequelae of this histamine release has been suggested as being fundamental to preventing the cytokine storm that may cause acute respiratory distress syndrome, leading to hypoxia, sepsis, organ failure, and ultimately death in the patient with COVID-19 [63]. Lower levels of ferritin, CRP, and procalcitonin in famotidine-treated patients in this study are compatible with the hypothesis that the drug may limit the abnormal excessive cytokine release from an uncontrolled immune activation. Wu et al. [62] used computational methods to predict structures of proteins encoded by the severe acute respiratory syndrome coronavirus 2 (SARS-CoV-2) genome and identified famotidine as one of the drugs most likely to inhibit the 3-chymotrypsin-like protease (3CL pro), which processes proteins essential for viral replication [61]. In addition, it is notable that certain unusual clinical aspects of COVID-19 could be explained by excessive histamine release and stimulation of the histamine- 2 receptor. First, along with early typical nonspecific viral symptoms of fever, sore throat, cough, headache, diarrhea, and myalgia, some patients with COVID-19 may experience anosmia, ageusia, and skin rashes including pruritis and urticarial symptoms [64,65]. All of these symptoms could be explained by histamine signalling. Mast cells located in the submucosa of the respiratory tract and in the nasal cavity represent a barrier of protection against microorganisms [66]. They play a role in inflammation development via release of multiple proinflammatory cytokines and chemokines [67]. Mast cells are known to be triggered by viruses [68], and it has been documented that they have the angiotensin-converting enzyme 2 receptor used by SARS-CoV-2 to gain entry to cells and replicate [69].

\section{Discussion}

Treatment with PPIs may be related to severe COVID-19 outcome, including increased mortality risk. Regular treatment with PPIs before hospitalization for COVID-19 has been associated with the risk of secondary infections and acute respiratory distress syndrome [18]. The highest risk is seen among individuals taking PPIs twice daily. The mechanisms by which this occurs are not fully understood.

PPI-induced hypochlorhydria with subsequent bacteria overgrowth in the upper gastrointestinal tract and microaspiration of gastric contents is known to be implied in PPI-associated pneumonia. Increased gastric alkalinity may also increase viral load in GI tract. In particular, coronaviruses were demonstrated to survive in individuals with more basic gastric $\mathrm{pH}$ levels, including those in the range created by the use of drugs like omeprazole and esomeprazole [70].

Another possible mechanism linking the PPI use with a major severity of the SARS-CoV-2 infection could be traced in the evidence that PPIs may lead to reduction of endothelial nitrous oxide levels through inhibition of dimethylarginine dimethylaminohydrolase enzymatic activity, which is responsible for clearance of asymmetric dimethylarginine, thereby decreasing nitrous oxide synthase activity [71]. This could be particularly relevant in consideration of the SARS-CoV-2's marked vascular tropism as widely demonstrated in the scientific literature [72].

For these reasons, even if PPIs are essential for the treatment of many disorders, their usage should be balanced considering the benefit to risk ratio.

In COVID-19 patients, we precautionary recommend to use the lowest possible dose of PPI for the control of the underlying condition even if this still is a matter of debate and controlledrandomized trials in SARS-COV-2 infections are required.

Interestingly, famotidine, a histamine-2 receptor (H2RA), has been associated with favorable clinical outcome in hospitalized patients with COVID-19, including lower in-hospital mortality, a lower composite of death and/or intubation, and lower levels of serum markers for serious disease [57,73].

Understanding the mechanisms by which host respiratory environment is more susceptible to SARS-COV-2 severe infection may better guide our approach to COVID-19 patients.

\section{References}

1. Wu Z, McGoogan JM. Characteristics of and important lessons from the coronavirus disease 2019 (COVID-19) outbreak in China: summary of a report of 72314 cases from the Chinese center for disease control and prevention. JAMA 2020;323:1239.

2. Sagnelli C, Celia B, Monari C, et al. Management of SARSCoV-2 pneumonia. J Med Virol 2021;93:1276-87.

3. Huang C, Wang Y, Li X, et al. Clinical features of patients infected with 2019 novel coronavirus in Wuhan, China. Lancet 2020;395:497-506.

4. Aziz M, Perisetti A, Lee-Smith WM, et al. Taste changes (Dysgeusia) in COVID-19: a systematic review and metaanalysis. Gastroenterology 2020;159:1132-3.

5. Aziz M, Haghbin H, Lee-Smith W, at al. Gastrointestinal predictors of severe COVID-19: syste- matic review and metaanalysis. Ann Gastroenterol 2020;33:1-16.

6. Perrotta, F., Corbi, G., Mazzeo, G., at al. COVID-19 and the elderly: insights into pathogenesis and clinical decision-making. Aging Clin Exper Res 2020;32:1599-608.

7. Iadevaia C, Perrotta F, Mazzeo G, at al. Incidental diagnosis of lung adenocarcinoma following coronavirus OC 43 severe pneumonia. Monaldi Arch Chest Dis 2020;90:1313.

8. Nigro E, Perrotta F, Polito R, et al. Metabolic perturbations and severe COVID-19 disease: Implication of molecular pathways. Int J Endocrinol 2020;2020:8896536.

9. Pottegard A, Kurz X, Moore N, et al. Considerations for pharmacoepidemiological analyses in the SARS-CoV-2 pandemic. Pharmacoepidemiol Drug Saf 2020;29:825-31.

10. Hariyanto TI, Kurniawan A. Metformin use is associated with reduced mor- tality rate from coronavirus disease 2019 (COVID-19) infection. Obes Med 2020;19:100290.

11. Pranata R, Permana H, Huang I, et al. The use of renin angiotensin system inhibitor on mortality in patients with 
coronavirus disease 2019 (COVID-19): a systematic review and meta-analysis. Diabetes Metab Syndr 2020;14:983-90.

12. Hariyanto TI, Kurniawan A. Statin therapy did not improve the in-hospital out- come of coronavirus disease 2019 (COVID-19) infection. Diabetes Metab Syndr 2020;14:1613-15.

13. Wang $\mathrm{CH}$, Li CH, Hsieh R, et al. Proton pump in- hibitors therapy and the risk of pneumonia: a systematic review and meta- analysis of randomized controlled trials and observational studies. Expert Opin Drug Saf 2019;18:163-72.

14. Vaezi MF, Yang YX, Howden CW. Complications of proton pump inhibitor therapy. Gastroenterology 2017;153:35-48.

15. Moayyedi P, Delaney BC, Vakil N, et al. The efficacy of proton pump inhibitors in nonulcer dyspepsia: a systematic review and economic analysis. Gastroenterology 2004;127:1329-37.

16. Luxenburger H, Sturm L, Biever P, et al. Treatment with proton pump inhibitors increases the risk of secondary infections and ARDS in hospital- ized patients with COVID-19: coincidence or underestimated risk factor? J Intern Med 2021;289:121-4.

17. Argenziano MG, Bruce SL, Slater CL, et al. Characterization and clinical course of 1000 patients with coronavirus disease 2019 in New York: retrospective case series. BMJ 2020;369:m1996.

18. Lee SW, Ha EK, Yeniova AO, et al. Severe clinical outcomes of COVID-19 associated with proton pump inhibitors: a nationwide cohort study with propensity score matching. Gut 2021;70:76-84.

19. Shin JM, Sachs G. Pharmacology of proton pump inhibitors. Curr Gastroenterol Rep 2008;10:528-34.

20. Trifan A, Stanciu C, Girleanu I, et al. Proton pump inhibitors therapy and risk of Clostridium difficile infection: systematic review and meta-analysis. World J Gastroenterol 2017;2 $3: 6500-15$.

21. Kanno T, Matsuki T, Oka M, et al. Gastric acid reduction leads to an alteration in lower intestinal microflora. Biochem Biophys Res Commun 2009;381:666-70.

22. Seto CT, Jeraldo P, Orenstein R, et al. Prolonged use of a proton pump inhibitor reduces microbial diversity: Implications for Clostridium difficile susceptibility. Microbiome 2014;2:42.

23. Darnell ME, Subbarao K, Feinstone SM, et al. Inactivation of the coronavirus that induces severe acute respiratory syndrome, SARS-CoV. J Virol Methods 2004;121:85-91.

24. Trottein F, Sokol H. Potential causes and consequences of gastrointestinal disorders during a SARS-CoV-2 infection. Cell Rep 2020;32:107915.

25. Xiao F, Tang M, Zheng X, et al. Evidence for gastrointestinal infection of SARS-CoV-2. Gastroenterology 2020;158:1831-3.e3.

26. Scialo F, Daniele A, Amato F, et al. ACE2: The major cell entry receptor for SARS-CoV-2. Lung 2020;198:867-77.

27. Lamers MM, Beumer J, van der Vaart J, et al. SARS-CoV-2 productively infects human gut enterocytes. Science 2020; 369:50-4.

28. Perrotta F, Matera MG, Cazzola M, Bianco A. Severe respiratory SARS-CoV2 infection: Does ACE2 receptor matter? Respir Med 2020;168:105996.

29. Hamming I, Timens W, Bulthuis ML, et al. Tissue distribution of ACE2 protein, the functional receptor for SARS coronavirus. A first step in understanding SARS pathogenesis. J Pathol 2004;203:631-7.

30. Zhang H, Liao YS, Gong J, et al. Clinical characteristics of coronavirus disease (COVID-19) patients with gastrointestinal symptoms: a report of 164 cases. Dig Liver Dis 2020;52:1076-9.

31. Sultan S, Altayar O, Siddique SM, et al. AGA Institute rapid review of the GI and liver manifestations of COVID-19, metaanalysis of international data, and recommendations for the consultative management of patients with COVID-19. Gastroenterology 2020;159:320-34.e27.

32. Hoffmann M, Kleine-Weber H, Schroeder S, et al. SARS-CoV2 cell entry depends on ACE2 and TMPRSS2 and is blocked by a clinically proven protease inhibitor. Cell 2020;181:271-80.

33. Dhar D, Mohanty A. Gut microbiota and Covid-19 - possible link and implications. Virus Res 2020;285:198018.

34. Boccia M, Aronne L, Celia B, et al. COVID-19 and coagulative axis: review of emerging aspects in a novel disease. Monaldi Arch Chest 2020;90:1300.

35. Monteleone G, Sarzi-Puttini PC, Ardizzone S. Preventing COVID-19-induced pneumonia with anticytokine therapy. Lancet Rheumatol 2020;2:e255-6.

36. Zhou J, Li C, Zhao G, et al. Human intestinal tract serves as an alternative infection route for Middle East Respiratory Syndrome coronavirus. Sci Adv 2017;3:eaao4966.

37. Eom, C.-S., Jeon, C. Y., Lim, J.-W et al. Use of acid-suppressive drugs and risk of pneumonia: a systematic review and metaanalysis. Can Med Assoc J 2011;183:310-9.

38. Laheij RJF, Sturkenboom MCJM, Hassing R-J, et al. Risk of community-acquired pneumonia and use of gastric acidsuppressive drugs. JAMA 2004;292:1955-60.

39. Altman KW, Waltonen JD, Hammer ND, et al. Proton pump $(\mathrm{H}+/ \mathrm{K}+-$ ATPase $)$ expression in human laryngeal seromucinous glands. Otolaryngol Head Neck Surg 2005;133:718-24.

40. Savarino V, Di Mario F, Scarpignato C. Proton pump inhibitors in GORD: An overview of their pharmacology, efficacy and safety. Pharmacol Res 2009;59:135-53.

41. Aybay C, Imir T, Okur H. The effect of omeprazole on human natural killer cell activity. Gen Pharmacol 1995;26:1413-8.

42. Zedtwitz-Liebenstein K, Wenisch C, Patruta S, et al. Omeprazole treatment diminishes intra- and extracellular neutrophil reactive oxygen production and bactericidal activity. Crit Care Med 2002;30:1118-22.

43. Lambert AA, Lam JO, Paik JJ, et al. Risk of communityacquired pneumonia with outpatient proton-pump inhibitor therapy: A systematic review and meta-analysis. PLoS One, 2015; 10:e 0128004.

44. Charpiat B, Bleyzac N, Tod M. Proton pump inhibitors are risk factors for viral infections: Even for COVID-19? Clin Drug Investig 2020;40:897-9.

45. Corsonello A, Lattanzio F, Bustacchini S, et al. Adverse events of proton pump inhibitors: potential mechanisms. Curr Drug Metab 2018;19:142-54.

46. Matera MG, Rogliani P, Bianco A, Cazzola M. Pharmacological management of adult patients with acute respiratory distress syndrome. Expert Opin Pharmacother 2020;21:2169-83.

47. Yoshida N, Yoshikawa T, Tanaka Y, et al. A new mechanism for anti-inflammatory actions of proton pump inhibitors - inhibitory effects on neutrophil-endothelial cell interactions. Aliment Pharmacol Ther 2000;14:7S4-81.

48. Savarino V, Marabotto E, Furnari M, et al. Latest insights into the hot question of proton pump inhibitor safety - a narrative review. Dig Liver Dis 2020;52:842-52.

49. Savarino V, Dulbecco P, Savarino E. Are proton pump inhibitors really so dan- gerous? Dig Liver Dis 2016;48:851-9.

50. Bhaskar S, Sinha a, Banach M, et al. Cytokine storm in COVID19-immunopathological mechanisms, clinical considerations, and therapeutic approaches: The reprogram consortium position paper. Front Immunol 2020;11:1648

51. Taghizadeh-Hesary F, Akbari H. The powerful immune system against powerful COVID-19: A hypothesis. Med Hypotheses 2020;140:109762. 
52. Kouhapey S, Shariati L, Boshtam M, et al. The molecular story of COVID-19; NAD+ depletion addresses all questions in this infection. Preprints 2020;2020030346.

53. Gommers LM, Hoenderop JG, Bindels RJ, de Baaij JH. Hypomagnesemia in type 2 diabetes: A vicious circle? Diabetes 2016;65:3-13.

54. Revathi R, Amaldas J. A clinical study of serum phosphate and magnesium in type II diabetes mellitus. Int J Med Res Health Sci 2014;3:808-12.

55. Roush GC, Sica DA. Diuretics for hypertension: A review and update. Am J Hypertens 2016;29:1130-7.

56. Chen XM, Li Y, Guo WL, et al. [Prevalence of laryngopharyngeal reflux disease in Fuzhou region of China].[Article in Chinese]. Zhonghua Er Bi Yan Hou Tou Jing Wai Ke Za Zhi 2016;51:909-9

57. Freedberg DE, Conigliaro J, Wang TC, et al. Famotidine use is associated with improved clinical outcomes in hospitalized COVID-19 patients: A propensity score matched retrospective cohort study. Gastroenterology 2020;159:1129-31.e3.

58. Janowitz T, Gablenz E, Pattinson D, et al. Famotidine use and quantitative symptom tracking for COVID-19 in nonhospitalised patients: A case series. Gut 2020;69:1592-7.

59. Bourinbaiar AS, Fruhstorfer EC. The effect of histamine type 2 receptor antagonists on human immunodeficiency virus (HIV) replication: Identification of a new class of antiviral agents. Life Sci 1996;59:365-70.

60. Malone RW, Tisdall P, Fremont-Smith P, et al. COVID-19: famotidine, histamine, mast cells, and mechanisms. Preprint ResearchSquare 2020;rs-30934.

61. Anand K, Ziebuhr J, Wadhwani P, et al. Coronavirus main proteinase (3CLpro) structure: Basis for design of anti-SARS drugs. Science 2003;300:1763-7.

62. Wu C, Liu Y, Yang Y, et al. Analysis of therapeutic targets for
SARS-CoV- 2 and discovery of potential drugs by computational methods. Acta Pharm Sin B 2020;10:766-88.

63. Kritas SK, Ronconi G, Caraffa A, et al. Mast cells contribute to coronavirus-induced inflammation: New anti-inflammatory strategy. J Biol Regul Homeost Agents 2020;34:9-14.

64. Eliezer M, Hautefort C, Hamel A, et al. Sudden and complete olfactory loss of function as a possible symptom of COVID-19. JAMA Otolaryngol Head Neck Surg 2020;146:674-75.

65. Giacomelli A, Pezzati L, Conti F, et al. Self-reported olfactory and taste disorders in SARS-CoV-2 patients: A cross-sectional study. Clin Infect Dis 2020;71:889-90.

66. Metcalfe DD, Baram D, Mekori YA. Mast cells. Physiol Rev 1997;77:1033-79.

67. Theoharides TC, Alysandratos KD, Angelidou A, et al. Mast cells and inflammation. Biochim Biophys Acta 2012;1822:21-33.

68. Marshall J S, Portales-Cervantes L, Leong E. Mastcell responses to viruses and pathogen products. Int $\mathrm{J}$ Mol Sci 2019;20:4241.

69. Caughey GH, Raymond W W, WoltersPJ. Angiotensin II generationby mast cell alpha- and beta-chymases. Biochim Biophys Acta 2000;1480:245-57.

70. Almario CV, Chey WD, Spiegel BMR. Increased risk of COVID-19 among users of proton pump inhibitors. Am J Gastroenterol 2020;115:1707-15.

71. Dhaun N, Webb DJ. Endothelins in cardiovascular biology and therapeutics. Nat Rev Cardiol 2019;16:491-502

72. Bianco A, Valente T, Perrotta F, et al. Remarkable vessel enlargement within lung consolidation in COVID-19 compared to AH1N1 pneumonia: a retrospective study in Italy. Heliyon 2021;7:e07112.

73. Mather JF, Seip RL, McKay RG. Impact of famotidine use on clinical outcomes of hospitalized patients with COVID-19. Am J Gastroenterol 2020;115:1617-23. 